\title{
Knowledge Acquisition Process for Intelligent Decision Support in Critical Health Care
}

\author{
Alexandra Cabral ${ }^{1}$, António Abelha ${ }^{2}$, \\ Maria Salazar ${ }^{1}$, César Quintas ${ }^{1}$, Filipe Portela ${ }^{3}$, José Machado ${ }^{2}$, \\ José Neves $^{2}$, Manuel Filipe Santos ${ }^{3}$ \\ ${ }^{1}$ Sistemas de Informação, Centro Hospitalar do Porto \\ Largo Prof. Abel Salazar, Porto, Portugal \\ ${ }^{1}$ \{alexandra, msalazar, cesar.quintas\}@chporto.min-saude.pt \\ ${ }^{3} \mathrm{CCTC},{ }^{3}$ Centro Algoritmi, \\ Universidade do Minho, Portugal, \\ ${ }^{2}\{$ abelha, jmac,jneves\}@di.uminho.pt \\ ${ }^{3}\{\mathrm{cfp}, \mathrm{mfs}\} @$ dsi.uminho.pt
}

\begin{abstract}
An efficient triage system is a good way to avoid some future problems and, how much quicker it is, more the patient can benefit. However, a limitation still exists, the triage system are general and not specific to each case. Manchester Triage System is a reliable known system and is focused in the emergency department of a hospital. When applied to specific patients' conditions, such the pregnancy has several limitations. To overcome those limitations, an alternative triage system, integrated into an intelligent decision support system, was developed. The system classifies patients according to the severity of their clinical condition, establishing clinical priorities and not diagnosis. According to the woman urgency of attendance or problem type, it suggests one of the three possible categories of the triage. This paper presents the overall knowledge acquisition cycle associated to the workflow of patient arrival and the inherent decision making process. Results showed that this new approach enhances the efficiency and the safety through the appropriate use of resources and by assisting the right patient in the right place, reducing the waiting triage time and the number of women in general urgency.
\end{abstract}

KEYWORDS: Triage; Emergency Department; Intelligent Decision Support Systems; Manchester Triage System; Critical Medicine. 


\section{INTRODUCTION}

Usually, when a patient arrives to medical emergencies is submitted to a triage process to determine the priority of treatments based on the severity of their clinical condition. The waiting time depends on the patient condition and if arrives by ambulance or not. Apart from this, it is a quick process that normally takes between 2 and 5 minutes.

In the hospital environment various types of triage systems are used. The most commonly used are those with five levels of severity, such as the Emergency Severity Index (ESI) (Tanabe, Gimbel, Yarnold, \& Adams, 2004), the Manchester Triage System (MTS) (Mackway-Jones, Marsden, \& Windle, 1997) and the Canadian Triage and Acuity Scale (CTAS) (Beveridge, Ducharme, Janes, Beaulieu, \& Walter, 1999). The main limitation with this type of scales is the lack of flexibility; normally they are only used in general emergency units.

Addressing to a specific type of emergency, in this case women who seek Gynecological and/or Obstetrics (GO) emergency care, was detected that the system they are using in the Hospital is not the most correct. They were assisted in the general Emergency Department (ED) of Hospital Geral de Santo António (HGSA) using the MTS, which hasn't been effective for GO due to the generality of the questions used for the triage.

In 2010, this type of care was transferred to Maternidade Júlio Dinis (MJD). MJD is a maternity hospital that provides care for women during pregnancy, childbirth and for newborn infants. Due to the fails detected in the triage system used, a new system was developed. However, the need for a triage system in MJD still exists because of the misclassification of non-urgent patients, when patient's severity is not identified at the triage stage, or if there is no accordance on what problems are non-urgent (Bianco, Pileggi, \& Angelillo, 2003; Hayden, Jouriles, \& Rosen, 2010; Rassin, Nasie, Bechor, Weiss, \& Silner, 2006; Wuerz, Fernandes, \& Alarcon, 1998).

Nowadays, in the ED of MJD, women who seek for GO emergency care (pregnant, non-pregnant, parous or primiparous) pass through a triage system that was specially developed for GO. However, some limitations remain due to:

- Limitations associated to the process of validating triage scales, because, even in developed countries, there are problems in conceptualizing validation methods (Twomey, Wallis, \& Myers, 2007);

- The maternal different symptoms that is possible to have and the difficult to represent them. These symptoms normally require a continuous and special evaluation. It should also be stressed that the limited budgets for health care make crucial to prioritize patients' needs and assist them with the most appropriate resources; 
- Having a single triage system can be not enough because sometimes the symptoms show a specific problem and, in other moment, a totally different disease. Joining that, having much information is difficult to disseminate this in the right time.

In order to support the decision-making process for a better healthcare in MJD distinguishing urgent and non-urgent patients an Intelligent Decision Support System (IDSS) was designed. The objective is, through the use of the knowledge discovery and data / text mining techniques predict the level of urgency and help to choose the better decision for each situation.

This IDSS will use the different data available, collected through the triage questionnaires, to help the emergency staff choosing the best decision to the women, in the moment they need the information.

To this approach the knowledge was obtained directly from the doctors' empirical and scientific experience to make the first version of decision models. Due to the particular gynaecological and/or obstetrics requests, other characteristics had been developed, namely a system that can increase patient safety for women in need of immediate care and help low-risk women avoid high-risk care, maximizing the use of resources.

Beyond the introduction, the chapter includes seven sections. The first one is related to the background knowledge and introduces the MJD, the MTS and the IDSS and its technological foundations. It also presents the actual context and the basics on the triage, how Manchester System works. The second section presents the gains with the changing of the triage local and the new triage process. The third section outlines the IDSS implemented for the GO purposes, the main objectives and the changes occurred. The fourth section introduces the Knowledge Discovery process designed to MJD. The sixth part of this paper presents some qualitative and quantitative results obtained so far. Finally, some discussions and conclusions are made.

\section{BACKGROUND AND RELATED WORK}

\section{Context}

MJD is part of Centro Hospitalar do Porto (CHP), together with Hospital de Santo António (HSA) and Hospital Maria Pia (HMP). CHP was created in 2007 and, prior to that, the three were separated entities. Women in need of urgent Care (GO) could attend the ED of either HGSA or MJD. Since the creation of CHP, women in such conditions are encouraged to attend the ED of MJD instead of HSA (ex-HGSA).

HSA has a general ED working with the MTS since 2000 and, to optimize the care provided to women in urgent or emergent situations, they are sent to the $\mathrm{ED}$ of MJD with a specific triage system that we developed, which is in use for six months, since January of 2010. During the last two years, were 
admitted into the MJD 36281 patients: in 2010, 18378 in 2010 and 17833 in 2011.

\section{Intelligent Decision Support System approach and technological foundations}

According to Turban (Turban, Aronson, \& Liang, 2005), a decision support system is an interactive, flexible and adaptable information system, developed to support a problem solution and to improve the decision making. These systems usually use artificial intelligence techniques and are based on prediction and decision models that analyse a vast amount of variables to answer a question.

The decision making process can be divided in five phases: intelligence, design, choice, implementation and monitoring (Turban, et al., 2005). To be an IDSS there exist some features that have to be accomplished. (Portela et al., 2010) The presence of an IDSS in an ED permits a better understanding of the real state of the patient and can improve the output results. It can help nurses giving the best care to the women, in the right time that they needs, improving the decision quality. Due to the complexity of the department, the first two phases (intelligence and design) of the decision making process will be very important.

The idea of creating an IDSS was motivated in the INTCare project that are in use in the Intensive Care Unit to predict outcome and organ failure to the patients admitted to the unit (Portela, et al., 2010). The system uses models induced through data mining techniques (Vilas-Boas, Santos, Portela, Silva, \& Rua, 2010) and a set of software agents to perform the tasks autonomously (Nealon \& Moreno, 2003; Santos et al., 2011). A similar approach will be followed in the GO area.

The interoperability with the other information systems is in charge of the AIDA system. AIDA (Agency for Integration, Diffusion and Archive of Medical Information) is a platform that consists of a Multi-Agent System (MAS) and overcomes difficulties in achieving uniformity of clinical systems, as well as medical and administrative complexity of different Hospital information sources (Abelha et al., 2003). "This platform features a proactive behaviour in its main functions: communication between heterogeneous systems, storage management and hospital information; response to requests in time; sending and receiving information from hospital sources like laboratories (labs) (medical reports, images, prescriptions, etc.)" (Duarte, Portela, Santos, António, \& José, 2011). AIDA establishes connection with all Systems of medical information: Electronic Health Records (EHR); Administrative Information System (AIS); Medical Information System (MIS); and Nursing Information System (NIS) (Machado, Abelha, Novais, \& Neves, 2010). Triage questionnaires also are implemented here, providing an online access. 


\section{Triage Systems}

Triage is a process developed with the agreement of a panel of experts based on decision rules and is an integral part of the modern ED (Moll, 2009). It is mostly supported by computerized information systems (Dong et al., 2006).

In the triage process, decision-making involves the interpretation, discrimination and evaluation of communication between the patient and the health care providers that are carrying out the triage process (Cronin, 2003).

Good decision making is crucial because patient outcome is greatly influenced by the initial assessment of the triage. The main method used in the triage process by the professional community in HSA is the MTS, which is described in the next section.

Additionally, it is explained why this system does not fit urgent and emergent situations for GO and is presented the system we developed for that purpose.

Obstetric triage has been one of the latest obstetric services to emerge throughout the last decade and hospitals have incorporated triage principles into the practice of GO (PSRS, 2008).

GO triage occurs in a women's hospital emergency unit, where a variety of GO conditions are presented, since labour assessment and assistance to common GO conditions. It is, therefore, of great importance to ensure that women who attend the triage unit are properly evaluated and that high-risk patients are accordingly treated, not just the mother, but also the fetus.

According to the severity level allotted to the patients in the ED, they are assigned to the appropriate observation area and the first observation should occur in a defined period of time.

The purposes of ED triage are described as "assigning the patient a place in queue, assigning an area or treatment room, predicting resource consumption and identifying non urgent patients who could be diverted to other, presumably less expensive venues" (Derlet, Kinser, Ray, Hamilton, \& McKenzie, 1995; Trzeciak \& Rivers, 2003). Likewise, triage for GO has the ability to screen, prioritize, and expedite GO conditions, and is used to rate the severity of women's condition and to optimize the resources available, for the resources of ED can be overused for non-urgent patients. This ability also lends itself to the emergent care skills needed in an obstetric triage unit as well as effective triaging (JáAngelini, 1999).

In conclusion, the main concerns in triage, either in GO or in other medical specialties are to improve the quality of care and reduce the risks arising from the waiting time to emergent care. Discrepancies in triage may lead to inappropriate use of resource and may contribute to both patient and staff dissatisfaction.

"If over-triage occurs, excessive resources are used for patients with nonurgent problems, resulting in excess costs and delays of care for the patients 
with more severe problems. If under-triage occurs, potentially sick patients may be triaged as non-urgent resulting in a clinically unsafe diversion from the ED." (Wuerz, et al., 1998).

\section{Manchester Triage System}

The Manchester Triage System (MTS) (Mackway-Jones, et al., 1997) is a five point triage scale used to triage patients presenting to the ED, which was introduced in the United Kingdom in 1996 and is now widespread, especially in Europe, and has been in use in HSA since 2000.

According to Wulp (van der Wulp, 2010), MTS can be defined as a system that contains a set of 52 flowcharts where each represent a patient complaint. Each flowchart contains discriminators which allow triage nurses to allocate a patient into an urgency category. An urgency category represents a maximum waiting time in the ED for the patient to be seen by a doctor. Patients who need to be seen by a doctor immediately are triaged in the most urgent category of the system, category red. When patients are triaged in the second urgency category, the orange one, they can wait up to ten minutes. The third category, yellow, contains patients who can wait up to sixty minutes. Patients triaged in category green can wait up to 120 minutes and patients triaged in category blue can wait up to 240 minutes. An important discriminator of the MTS is pain. Pain can be assessed with the systems' pain ruler. This instrument consists of a visual analogue scale, a pain behaviour tool and a verbal descriptor scale. Pain assessments can direct a nurse immediately to the patient's level of urgency. Although the MTS is a reliable system of triage in the ED, well suited for general emergency situations, in particular cases like the GO care, its use is unsatisfactory due to the generalization of the questions it uses for classifying patients' clinical severity status. This system workflow confines to a number of presenting problems with associated flowcharts and supporting documentation. It has five levels of classification. Table 1 presents the five levels of classification of the MTS and the corresponded recommended waiting times for patients.

Table 1. - MTS levels and recommended waiting times (Services, 2003)

\begin{tabular}{|cc|}
\hline Levels & Patient should be seen by provider within \\
\hline 1 - Immediate & 0 minutes \\
2 - Very urgent & 10 minutes \\
3 - Urgent & 60 minutes \\
$4-$ Standard & 120 minutes \\
5 - Non-urgent & 240 minutes \\
\hline
\end{tabular}


The benefits of the MTS were examined and considered to overcome other systems (Cronin, 2003). Some advantages were identified, highlighting the fact that the MTS is a method of triage internationally recognized, reliable and professionally evaluated and successfully used in a number of different health care systems.

Although the literature states that the MTS is a suitable evidence-based triage method and could be expanded to include other EDs of band one hospitals (Cronin, 2003), it is not suitable to ED for GO, as experienced in HSA. Discussions arose that led to the conclusion that the criteria document that supports the MTS system did not satisfy the requirements for emergency care in GO.

MTS is simple enough to allow a quickly assess of the patients' clinical condition and is applicable for the wide-range population with a large variability in signs and symptoms in the emergency care setting (Moll, 2009). Therefore, its use is more suitable for general medical emergencies and not the specific emergencies of women in need of GO medical assistance.

Furthermore, the discriminatory power of MTS is not equal for medical and surgical specialties, which may be related to the character of its natural discriminators (Martins, De Castro Dominguez Cuña, \& Freitas, 2009).

Triage is a process developed with the agreement of a panel of experts based on decision rules and is an integral part of the modern ED (Moll, 2009). It is mostly supported by computerized information systems (Dong, et al., 2006).

In the triage process, decision-making involves the interpretation, discrimination and evaluation of communication between the patient and the health care providers that.

Based on a study done with the objective to evaluate Reliability and validity of the Manchester Triage System in a general emergency department, (Van Der Wulp, Van Baar, \& Schrijvers, 2008), was possible to prove that MTS isn't focused in this environment. Through the results obtained so far, was possible observe that "The reliability of the MTS is not influenced by nurses' work experience. Undertriage mainly occurs in the MTS categories orange and yellow. The MTS is more sensitive for children who need immediate or urgent care than for other patients in the emergency department." (Van Der Wulp, et al., 2008). Having in consideration that, in general, all of our patients are adult women, the probability of the triage result be incorrect is considerable. This was one of the reasons that justified the development of a specific triage system to GO.

TRIAGE SYSTEM FOR GYNECOLOGY AND OBSTETRICS IN MJD 
Prior to the creation of CHP, pregnant women and women in need of urgent or emergent care, at first, were treated as a normal patient and were admitted in HGSA and MJD for emergency situations. In HGSA, the MTS had been used since 2000 and doesn't discriminate the origin sources, i.e., the type and group the patient health problem.

However, the healthcare professionals realized that the MTS was not well suited to the GO service due to the degree of generalization of the guidelines. Moreover, more than $40 \%$ of the cases were not emergencies, which would translate in a misuse of resources, i.e., women made triage without this necessity, delaying the triage time for the other patients.

Accordingly, a triage system specific for GO was developed, where the guidelines are focused on that particular type of patients / condition, i.e. pregnant or parous women. After the creation of CHP, the GO emergencies were transferred to MJD and the women were attended according the results obtained by the new triage system.

As depicted in the graph of the Figure 1, a decrease of $90 \%$ in the GO emergencies episodes has been achieved. This graph compares the triage discharge of the last three years $(2009,2010$ and 2011), taking into account the place where the women have been triaged.

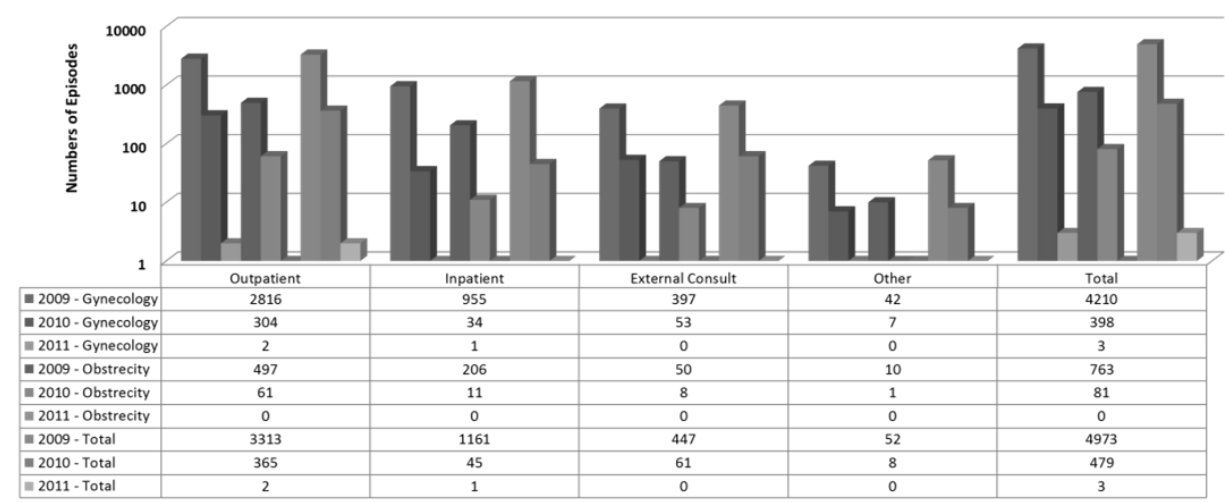

Figure 1. GO Episodes in CHP

Was developed an effective triage model that meets the needs of the ED for GO, in particularly a system that can increase patient safety for women in need of immediate care (proving) and help low-risk women avoid high-risk care, maximizing the use of resources.

The goal of this system is, classify patients according to the severity of their clinical condition, establishing clinical priorities and not diagnosis.

The triage is done by specialized physicians and is based on a set of predefined questions in the form of rules of a decision tree. According to the result, the IDSS indicates whether the patient should be sent to an urgent 
(URG) or normal consultation (ARGO - Outpatient Clinic). Women in need of urgent care (e.g. women who arrive by ambulance in very serious conditions) are immediately assisted, without any triage.

Before, in MJD, women were admitted in the order of their arrival, not differentiating the cases that required immediate or intermediate assistance and the non-urgent cases, taking some urgent women waiting much time for triage and non-urgent cases quickly attended. In HGSA they were classified with the MTS.

The IDSS brought some improvement in healthcare, essentially because:

- Contributes to the decrease of clinical errors;

- Provides cost-effective and proper care;

- Improves client satisfaction.

Moreover, by transferring all the GO emergencies to MJD and correctly assessing their severity, it also contributes for reducing overcrowding of ED (Johnson, Myers, Wineholt, Pollack, \& Kusmiesz, 2009; Olshaker, 2009; Solberg, Asplin, Weinick, \& Magid, 2003)

When women arrive at the MJD requiring urgent observation, and before any admission paperwork, a physician makes the triage using the system. It indicates if the patient should be assisted with urgency or be sent to a normal first medical appointment.

In opposition to the MTS, triage is done by physicians and not by nurses. In general ED triage, nurses usually gather the clinical information and make the triage decision.

However, tests in MJD showed a lack of agreement in triage assessment between nurses and doctors. Moreover, a study showed that the triage decisions physicians make are significantly different when they have and take the opportunity to visually evaluate patients (Brillman, Doezema, Tandberg, Sklar, \& Skipper, 1997).

Accordingly, a visual assessment can enhance the doctors' intuitive ability to quickly distinguish a sick from a not-so-sick patient. In conclusion, close supervision by a doctor is essential because of the significant risks involved (Bygaryventolini \& Ranneiger, 2003).

\section{REAL-TIME DECISION PROCESS}

The workflow of Figure 2 describes the real-time decision process in ED whenever a woman is admitted into MJD.

When a woman arrives to the MJD, the type of transport used is very important. If she was transported by an ambulance then the woman will be conducted to a room for immediate assistance. When another transport is used, the condition of the woman is evaluated to determine if an urgent observation is required, this will be done, through a quick and urgent 
observation, and if, the problem is severe, she also will be immediately assisted. Only, if an urgent observation weren't required, the woman will be inserted in the triage process. At this moment, she needs to wait a few seconds / minutes to be called to the triage room. In the triage room, she will answer to a questionnaire provided by the emergency department staff. This questionnaire is the specific to GO.

Like said before, this is a quick process, only a few important questions will be answered. This process is totally electronic and is supported by the AIDA platform. The questionnaire is available in AIDA where, after be opened, the nursing staff follow a set of pre-defined questions organized in groups, with defined options or possibility to write some observations, that allow a first perception of the real woman condition.

Immediately after the questionnaire be completed, the system will consult a set of models that are suitable for the actual case and try to define the woman condition anticipating the urgency degree of attendance, i.e., emergent, urgent or non-urgent.

According to this result, the nurses will decide what do and then conduct the woman into a specific room. In the case of a non urgent situation a consultation will be schedule. This consultation can be of two types: urgent or normal, according to its urgency.

The results obtained and decisions made, will be stored for further improvement of the decision models. 


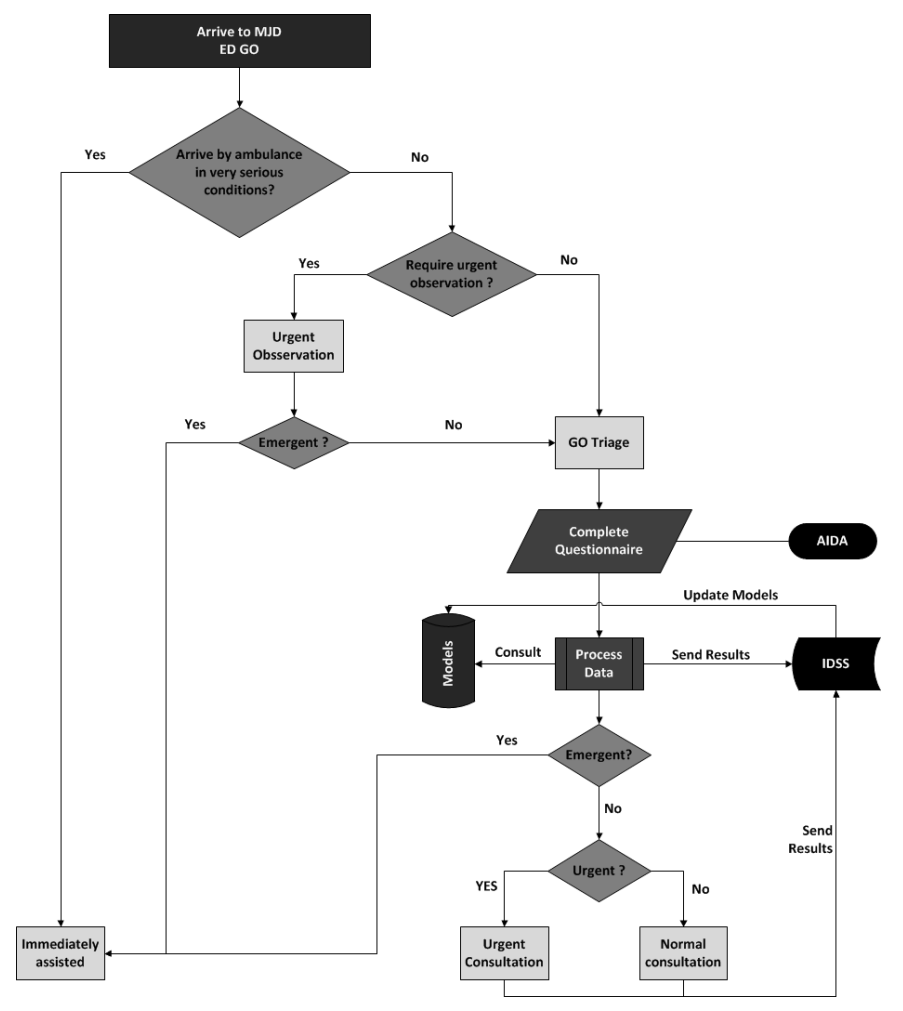

Figure 2. GO workflow

In the future, the results of the questionnaires and consultations associated to the decisions made during the experimental period will be used to create prediction models in order to accurately discriminate the urgent cases, dividing the actual urgent class in two subclasses: urgent and possibly urgent. The last type of patients will be directed to a normal consultation. During the waiting time they will be in observation and if something happens aggravating the clinical condition, the priority will be changed to urgent and the patient will be immediately assisted.

\section{KNOWLEDGE DISCOVERY PROCESS}

The system implemented here, was based in the Data Acquisition System and Knowledge Discovery Database (KDD) that was developed in the ICU (Portela et al., 2011; Portela, Santos, Silva, Machado, \& Abelha, 2011). Although the objectives and the data are different the concept is the same: to have all the data and results available online and in real-time. Before the KDD process, the questionnaires data should be collected. After the questionnaire 
be filled, an agent is responsible to read the results, which are in an xml format, interpret them and store it.

Figure 3 presents the KDD process and how the results obtained at the end of each task can be used. The process starts with the raw data acquired from the sources and culminates with the new knowledge. The Extract, Transform and Load (ETL) process involves the three initial phases and consists in the extraction of data from the sources, transformation of these data and loading the final data into the data warehouse, i.e. to prepare the data to be used by data mining algorithms.

The database is populated with data from four sources: triage (questionnaires and decisions taken), Electronic Health Records for this patient, and if exist, the therapeutic plan and Lab Results. The data will be selected to the data warehouse to be processed or transformed, depending of the use goal to each variable defined in Data Mining (DM) and Text Mining (TM) models. After this task, the data will be stored in database and is prepared for the creation of DM/TM Mining Models. The data obtained will be used to train and induce the models corresponding to GO guidelines useful to optimize decisions. These are the decision models that will be available in the triage after the questionnaires were completed. Finally all models will be evaluated and, the knowledge obtained will be presented in IDSS.

The data processed and treated will be stored in the database and will be prepared to be used by the system to predict future states of patients and anticipate health problems.

All data acquisition, pre-processing and selection tasks will be supported by intelligent agents. Now, the system is using a traditional approach to predict the results. New data mining / text mining models are being studied.

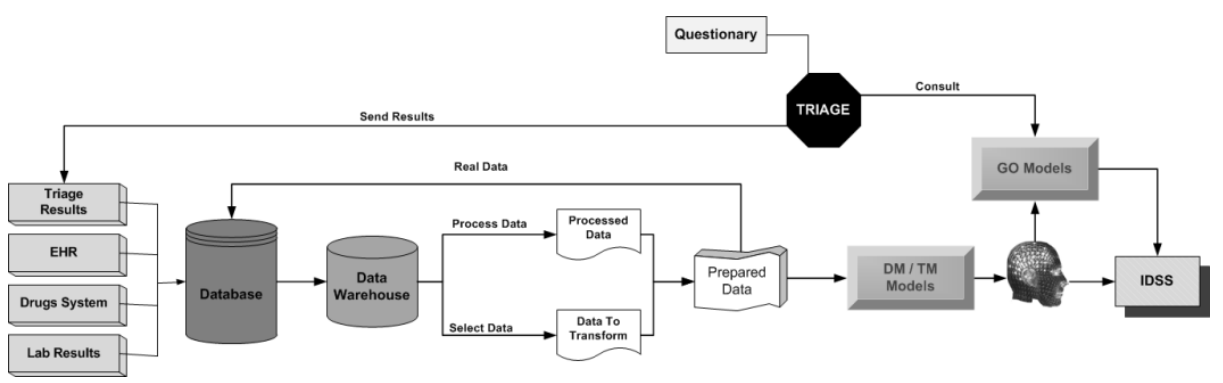

Figure 3. GO Knowledge Discovery from Database Process

\section{RESULTS}

The system is working since January of 2010. In two years, 36281 women have been triaged by the system. Figure 4 shows the distribution of the 
possible causes pointed by the patients as the motivation to come to MJD. As reported, the number of women is similar (18378 in 2010, 17833 in 2011), where around $85 \%$ were or not pregnant, the other $15 \%$ are women that need to be observed in other units like Institute of Gynecology and Obstetrics (IGO) or maybe were pregnant. Figure 4 also shows the average of the waiting time to triage. This time decreased in all groups, a general reduction about $20 \%$ was observed.

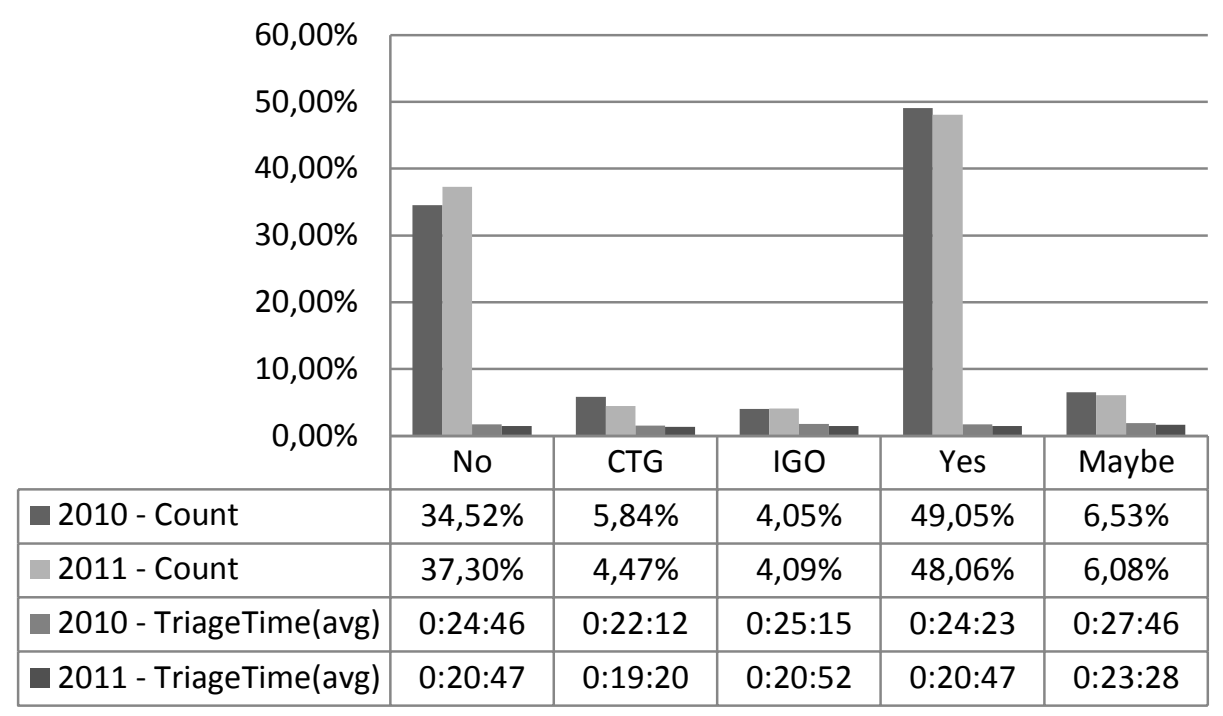

Figure 4. Woman Group vs. Triage time average

Figure 5 shows the distribution of the population by the three possible categories of the triage: non-urgent (ARGO), urgent (URG) and emergency (EMERG). The differences between the last two years are significant. The percentage of URG cases decreased $17 \%$, the ARGO increased $10 \%$ and EMERG increased $+200 \%$. More than $50 \%$ of the cases (9221) were classified as non-urgent, which means that those women were sent to a normal first medical appointment, reducing ED overcrowding and contributing to a better efficiency.

Without this system, all women would have been assisted as urgent, according to their time of arrival, not taking the severity (or lack of) into account to evaluate their clinical condition.

The effects of this scenario would be the misuse of resources and potentially more urgent cases could be assisted after the less urgent. It is also worth noting that, in two years, more than 35000 women was assisted in MJD, contributing to less crowding in the ED of HSA. 


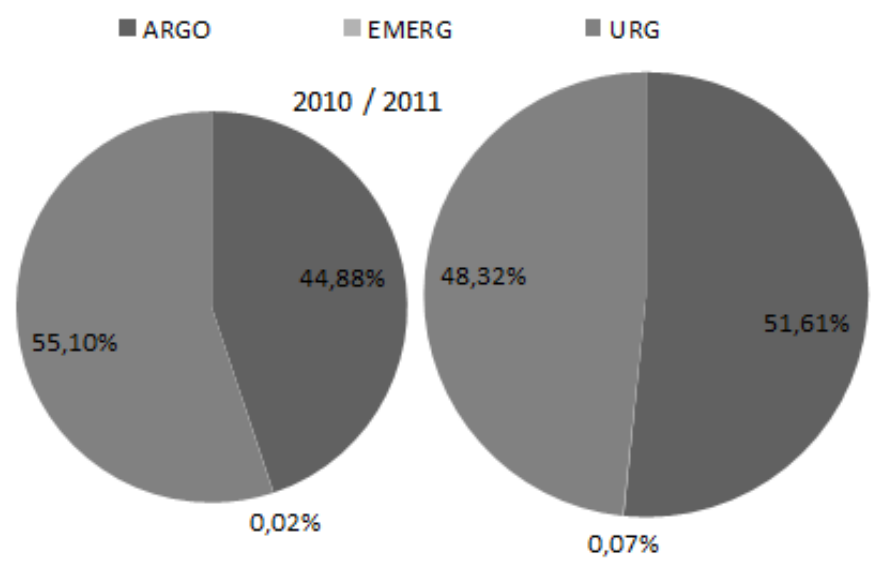

\begin{tabular}{|c|r|r|r|r|}
\hline RESULT & \multicolumn{1}{|c|}{$\mathbf{2 0 1 0}$} & \multicolumn{1}{c|}{$\mathbf{2 0 1 1}$} & \multicolumn{1}{c|}{ Dif } & Total \\
\hline ARGO & 8265 & 9221 & $10,37 \%$ & 17486 \\
\hline EMERG & 4 & 12 & $200,00 \%$ & 16 \\
\hline URG & 10146 & 8633 & $-17,53 \%$ & 18779 \\
\hline Total & 18415 & 17866 & $-3,07 \%$ & 36281 \\
\hline
\end{tabular}

Figure 5. Results of the triage system

\section{DISCUSSION}

The actual work methodology and the system used in MJD is different, and even though it is a two-level system, in opposition to the prior system with a five-scale (MTS). The new system has proven to be more effective and brought benefits in healthcare of GO emergencies, in comparison to the previous one, where the attendance was done by arrived order and however sometimes was analysed the clinical risk. With this system, the probability of women needing for an emergency treatment be quickly attended is higher. By other side, the specificity of the questionnaires gives a better idea of the women condition, i.e., discriminating better the cases triaged, being fairer with women in worst conditions and assigning the right triage category. Patient priority is defined by the classes' urgent or first medical appointment. Although it is not a class, emergent cases have the highest priority and are sent immediately for the observation room, with no need for triage. With this strategy it is guaranteed that resources are optimally used and that women are assisted accordingly to the urgent degree of their medical condition, which has been pointed as major issues in triage systems. Other results will be determined according the triage questionnaire answers.

The modifications introduced in the triage, increased the number of the first consults, reducing the waiting list, and improving the triage waiting time at the same time. These results corroborate the decision to direct women triage 
to a specific service (MJD), benefited the triage of the all patients, because, with this modification, less 4970 women a year, appear in HSA urgency.

\section{CONCLUSION \& FUTURE WORK}

This chapter introduced the foundations for the deployment of an IDSS in the triage of Gynaecological and/or Obstetrics emergency department of Centro Hospitalar of Porto, Portugal. Important changes were made in the triage, a new triage questionnaire, were developed, which is a good way to obtain data to the IDSS, at the same time, makes a good representation of the woman condition. With this system the professionals can better assist the patients according to their degree of urgency i.e. if a case is really urgent (emergent) is because is too danger for the patient health and she should be treated instantly. If the case is not emergent, the triage system will be activated and as quickly as possible the woman condition will be "categorized". In the last year the waiting times decreased significantly to a level lower than the obtained by the Manchester triage system.

The changes made also are important to the main hospital, i.e., the number of emergency cases in HSA reduced significantly. Women needing for urgent care normally are no more directed for the general emergency department.

Patients are categorized into three different degrees: Emergent, Urgent or Normal. They will be observed / attended according to the level of risk. The IDSS help the nursing staff to make the better decision according to decision rules that in the future will be integrated in the prediction models.

These changes can prove that, the use of clinical knowledge of this specific area allow for a more suited system to this type of users, contrary to what it happened previously.

The results obtained so far also proved that the introduction of specialized triage system improves the decision and benefit the patients. The improvements done turned CHP eligible to receive a money prize, by the Health Ministry, as a performance bonus to ensuring greater accessibility to special care.

Future work includes the use of the data collected to optimize the rules and the data mining models as a way to improve the IDSS performance and to generate more accurate knowledge.

ACKNOWLEDGMENTS: The authors would like to express their gratitude to FCT (Foundation of Science and Technology, Portugal), for the financial support through the contract INTCare - PTDC/EIA/72819/2006. The work of Filipe Portela was supported by the grant SFRH/BD/70156/2010 from FCT.

\section{REFERENCES}


Abelha, A., Machado, M., Santos, M., Sollari, A., Rua, F., Paiva, M., et al. (2003). Agency for Archive, Integration and Diffusion of Medical Information. Proceeding of AIA.

Beveridge, R., Ducharme, J., Janes, L., Beaulieu, S., \& Walter, S. (1999). Reliability of the Canadian Emergency Department Triage and Acuity Scale: Interrater Agreement. Annals of Emergency Medicine, 34(2), 155-159.

Bianco, A., Pileggi, C., \& Angelillo, I. F. (2003). Non-urgent visits to a hospital emergency department in Italy. Public Health, 117(4), 250-255.

Brillman, J. C., Doezema, D., Tandberg, D., Sklar, D. P., \& Skipper, B. J. (1997). Does a physician visual assessment change triage?* 1 . The American journal of emergency medicine, 15(1), 29-33.

Bygaryventolini, M. D., \& Ranneiger, M. D. (2003). Avoiding the pitfalls of obstetric triage. $O B G$ MANAGEMENT.

Cronin, J. G. (2003). The introduction of the Manchester triage scale to an emergency department in the Republic of Ireland. Accident and Emergency Nursing, 11(2), 121-125.

Derlet, R. W., Kinser, D., Ray, L., Hamilton, B., \& McKenzie, J. (1995). Prospective identification and triage of nonemergency patients out of an emergency department: a 5-year study. Annals of emergency medicine, 25(2), 215-223.

Dong, S. L., Bullard, M. J., Meurer, D. P., Blitz, S., Ohinmaa, A., Holroyd, B. R., et al. (2006). Reliability of computerized emergency triage. Academic Emergency Medicine, 13(3), 269-275.

Duarte, J., Portela, F., Santos, M. F., Abelha, A., \& Machado, J. (2011). Electronic Health Record in Dermatology Service. Paper presented at the HCist 2011 - International Workshop on Health and Social Care Information Systems and Technologies, Algarve, Portugal.

Hayden, S. R., Jouriles, N. J., \& Rosen, P. (2010). Requiem for "Non-Urgent" Patients in the Emergency Department. The Journal of Emergency Medicine, 38(3), 381-383.

Johnson, M., Myers, S., Wineholt, J., Pollack, M., \& Kusmiesz, A. L. (2009). Patients who leave the emergency department without being seen. Journal of Emergency Nursing, 35(2), 105-108

JáAngelini, D. (1999). Obstetric triage: the realm of the midwife part 2. Journal of Nurse-Midwifery, 44(6), 536

Machado, J., Abelha, A., Novais, P., \& Neves, J. (2010). Quality of service in healthcare units. International Journal of Computer Aided Engineering and Technology, 2(4), 436-449.

Mackway-Jones, K., Marsden, J., \& Windle, J. (1997). Emergency triage: Manchester triage group: BMJ.

Martins, H. M. G., De Castro Dominguez Cuña, L. M., \& Freitas, P. (2009). Is Manchester (MTS) more than a triage system? A study of its association with mortality and admission to a large Portuguese hospital. Emergency Medicine Journal, 26(3), 183.

Moll, H. A. (2009). Challenges in the validation of triage systems at emergency departments. Journal of clinical epidemiology.

Nealon, J. L., \& Moreno, A. (2003). Agent-Based Applications in Health Care. Applications of Software Agent Technology in the Health Care Domain, 3-18.

Olshaker, J. S. (2009). Managing emergency department overcrowding. Emerg Med Clin North Am, 27, 593-603.

Portela, F., Gago, P., Santos, M. F., Silva, A., Rua, F., Machado, J., et al. (2011). Knowledge Discovery for Pervasive and Real-Time Intelligent Decision Support in Intensive Care Medicine. Paper presented at the KMIS 2011- International Conference on Knowledge Management and Information Sharing.

Portela, F., Santos, M., Vilas-Boas, M., Rua, F., Silva, Á., \& Neves, J. (2010). Real-time Intelligent decision support in intensive medicine. Paper presented at the KMIS 2010- International Conference on Knowledge Management and Information Sharing.

Portela, F., Santos, M. F., Silva, Á., Machado, J., \& Abelha, A. (2011). Enabling a Pervasive approach for Intelligent Decision Support in Critical Health Care. Paper presented at the HCist 2011 International Workshop on Health and Social Care Information Systems and Technologies.

PSRS. (2008). The Pennsylvania Patient Safety Advisory. $V$, 110. Retrieved from https://www.ecri.org/Documents/PA_PSRS/2008.09_Advisory.pdf

Rassin, M., Nasie, A., Bechor, Y., Weiss, G., \& Silner, D. (2006). The characteristics of self-referrals to ER for non-urgent conditions and comparison of urgency evaluation between patients and nurses. Accident and Emergency Nursing, 14(1), 20-26.

Santos, M. F., Portela, F., Vilas-Boas, M., Machado, J., Abelha, A., \& Neves, J. (2011). INTCARE - Multiagent approach for real-time Intelligent Decision Support in Intensive Medicine. Paper presented at the 3rd International Conference on Agents and Artificial Intelligence (ICAART), Rome, Italy. 
Services, U. S. D. o. H. H. (2003). Emergency Severity Index - Five-level Triage Systems. Retrieved 20/01/2011, 2011, from http://www.ahrq.gov/research/esi/esitab2-2.htm

Solberg, L. I., Asplin, B. R., Weinick, R. M., \& Magid, D. J. (2003). Emergency department crowding:: Consensus development of potential measures. Annals of emergency medicine, 42(6), 824-834.

Tanabe, P., Gimbel, R., Yarnold, P. R., \& Adams, J. G. (2004). The Emergency Severity Index (version 3) 5-level triage system scores predict ED resource consumption. J Emerg Nurs, 30(1), 22-29.

Trzeciak, S., \& Rivers, E. P. (2003). Emergency department overcrowding in the United States: an emerging threat to patient safety and public health. British Medical Journal, 20(5), 402.

Turban, E., Aronson, J. E., \& Liang, T.-P. (2005). Decision Support Systems and Intelligent Systems (7 ed.): Prentice Hall.

Twomey, M., Wallis, L. A., \& Myers, J. E. (2007). Limitations in validating emergency department triage scales. Emergency Medicine Journal, 24(7), 477.

van der Wulp, I. (2010). Reliability and validity of emergency department triage systems.

Van Der Wulp, I., Van Baar, M. E., \& Schrijvers, A. J. P. (2008). Reliability and validity of the Manchester Triage System in a general emergency department patient population in the Netherlands: results of a simulation study. Emergency Medicine Journal, 25(7), 431.

Vilas-Boas, M., Santos, M. F., Portela, F., Silva, Á., \& Rua, F. (2010). Hourly prediction of organ failure and outcome in intensive care based on data mining techniques. Paper presented at the 12th International Conference on Enterprise Information Systems.

Wuerz, R., Fernandes, C., \& Alarcon, J. (1998). Inconsistency of emergency department triage. Annals of emergency medicine, 32(4), 431-435. 